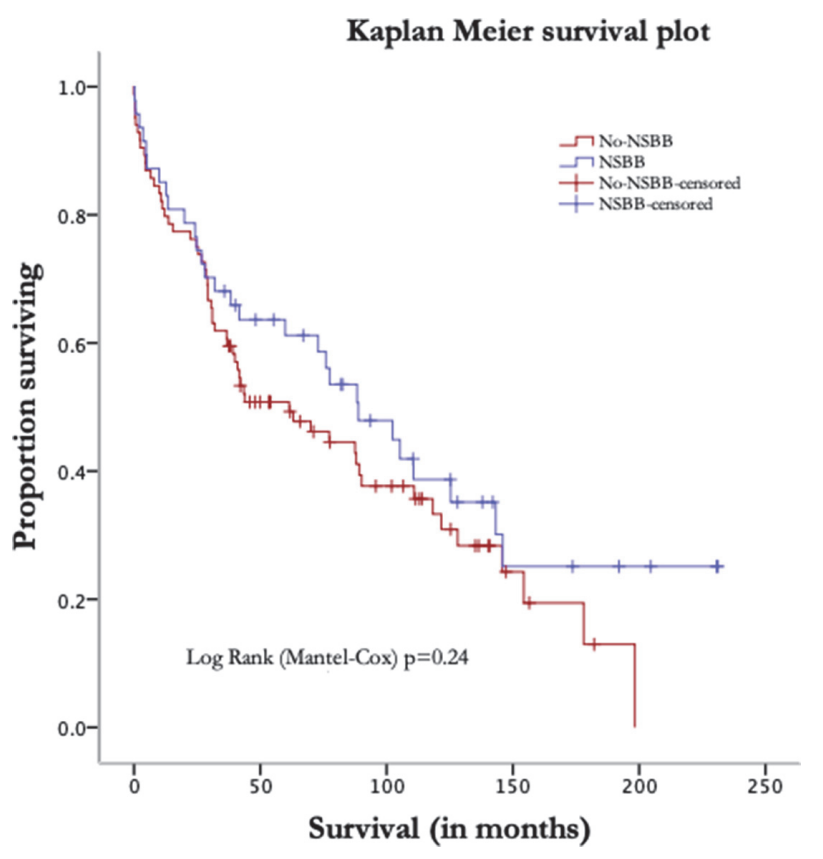

Abstract PWE-31 Figure 1 A graphical illustration of the survival times in the two groups is shown in the Kaplan-Meier plot

significant difference in survival between the two groups $(p=0.24)$ as seen in figure 1 . The adjusted hazard risk analysis (0.75 [95\%CI 0.51, 1.11], $p=0.15)$ suggested weak evidence of a difference in survival between the two groups. Median survival times were slightly longer in the NSBB group, 88.8 months (95\%CI 53.2,124.4) compared with No-NSBB group 61.4 months $(95 \%$ CI 26.6, 96.2); and the risk of death at any time lower. TIPSS, was performed in 6 patients of NoNSBB and 4 with NSBB group. Two patients received liver transplant, both in the no-NSBB group. Diuretic intractable ascites $(p=0.06)$, MELD $(p=0.018)$ and frequency LVP sessions $(p<0.001)$ were all independent predictors of mortality. Conclusions Our study demonstrates that NSBB is safe in cirrhotic patients with severe ascites, and it is not associated with detrimental outcome. There is need for a randomised controlled trial to draw conclusive evidence.

\section{PWE-32 THE EFFECTIVENESS OF HEPATOCELLULAR CARCINOMA SURVEILLANCE DURING COVID-19}

${ }^{1}$ William Cunliffe*, 1,2Thomas Pembroke. ${ }^{1}$ Cardiff Medical School, Cardiff, UK; ${ }^{2}$ Department of Gastroenterology and Hepatology, University Hospital of Wales, Cardiff, UK

\subsection{6/gutjnl-2021-BSG.222}

Introduction The incidence of hepatocellular carcinoma (HCC) in Wales is rising. We aimed to evaluate the impact of the COVID-19 pandemic on the South Wales HCC multi-disciplinary meeting (MDM) referrals and patient management.

Methods Baseline patient characteristics at MDM and treatment information was collated for index HCC discussion from the electronic health record. The year of the COVID period (CP) $01 / 03 / 2020-28 / 2 / 2021$, was compared to the prepandemic period (PP) 01/03/2018-29/02/2020. To determine significance, a $\mathrm{Chi}^{2}$ analysis or Fisher's Exact test were used where appropriate.

Results There was a 3-fold increase in index HCC diagnoses in South Wales MDM between 2002 and 2018. 803 patients were discussed from March 2018-21; follow up (372) and non-HCC (90) cases were excluded. There were $245 \mathrm{PP}$ and 96 CP index HCC diagnoses; a $22 \%$ reduction in annual index HCC diagnoses in the COVID period. The absolute number of HCCs detected by surveillance remained consistent at 33 per annum in $\mathrm{PP}$ and $\mathrm{CP}$ periods $(27 \% \mathrm{v} 34 \%$ respectively). During CP the proportion of patients offered best supportive care and undergoing further investigation increased (47 to $56 \%$ and 1.2 to $13.5 \%$ respectively) whilst all anti-cancer interventions fell (figure 1): ablation/surgery $(22$ to $15.6 \%)$, chemo-embolisation (21.2 to $11.5 \%)$ and systemic anti-cancer therapies (8.2 to $3.1 \%, \mathrm{p}<0.0001)$. However, during $\mathrm{CP}$ a greater proportion of those on HCC surveillance received curative therapies compared to HCC detected in individuals with cirrhosis outside of screening (27.3 vs. $8.5 \%$, $\mathrm{p}=0.03$ ). Median time from point of suspicion of HCC to

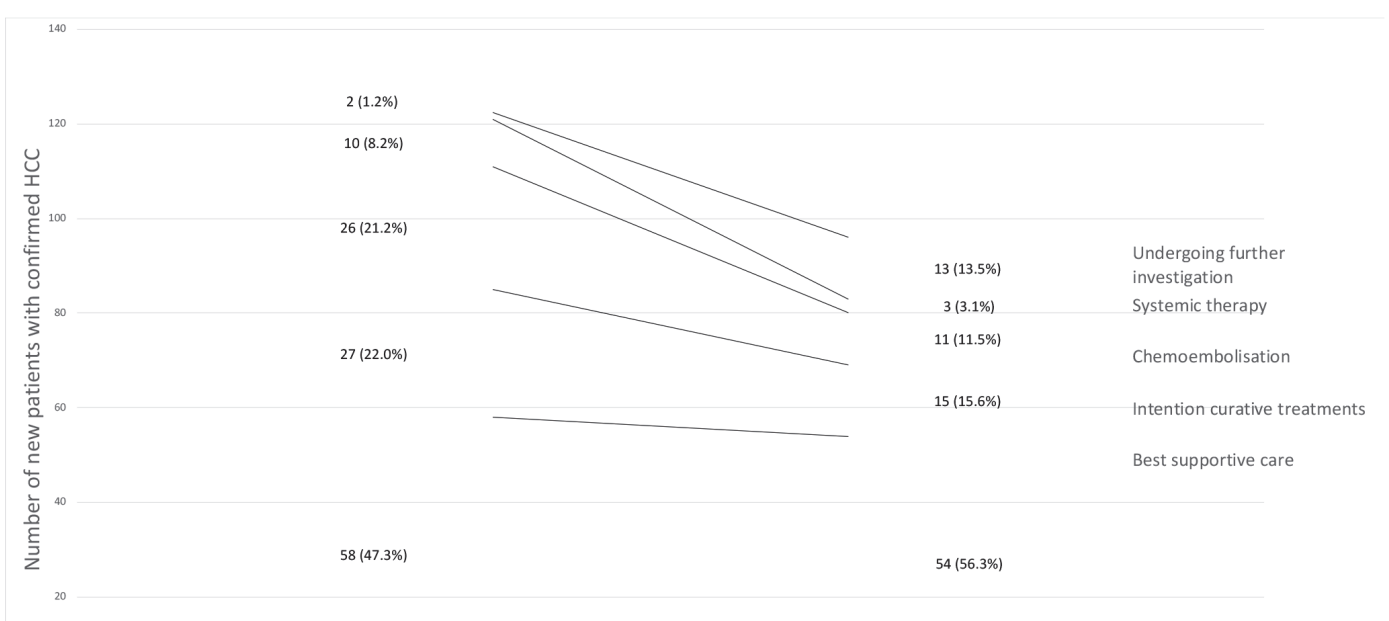

Pre-pandemic period 12-month average

COVID period

Abstract PWE-32 Figure 1 Treatment outcomes for HCC MDM discussion in South Wales in the Pre-pandemic and COVID periods 
MDM and MDM to first definitive treatment reduced slightly during the CP $(43 \quad \mathrm{v} \quad 40.5$ days and $56 \quad \mathrm{v} \quad 55$ days respectively).

Conclusions The COVID-19 pandemic had a significant impact on referrals to the HCC MDM, yet HCC surveillance identified the same number of hepatomas. HCC identified by surveillance in the pandemic were more amenable to radical therapies. The fall in the proportion of patients receiving both curative and palliative therapies reflects the fall in incidental treatable HCC during the COVID period. A relatively high proportion of patients received best supportive care in all three years; reflecting an older population with high burden of comorbidities in South Wales linked to socio-economic deprivation. Median waiting times are in excess of the Welsh Government's target of 62 days, suggesting that the current diagnosis and referral process requires revision and resources to expedite therapies.

\section{PWE-33 RITUXIMAB INITIATION, PRESCRIBING AND HEPATITIS B REACTIVATION: RETROSPECTIVE FIVE-YEAR REVIEW}

Zillah Cargill*, Nicola Grasso, George Bird. Maidstone Hospital, Maidstone and Tunbridge Wells NHS Trust, Kent, UK

\subsection{6/gutjnl-2021-BSG.223}

Introduction Hepatitis B reactivation is a potentially life-threatening complication of immunosuppression in patients with serological evidence of current or past exposure to Hepatitis B Virus (HBV). Rituximab is a widely used B-cell depleting medication widely used to treat haematological cancers and rheumatological disease. The British Society of Rheumatology, British Society of Haematology and the European Association of Study of the Liver guidelines all recommend patients must all be assessed for HBV before rituximab initiation. This is a single-centre five-year retrospective review of our Trusts Rituximab prescribing and HBV screening.

Methods We included all adult patients receiving rituximab between 1st October 2015 and 30th September 2020 at our Trust. Patients were identified using hospital pharmacy Rituximab prescribing logs. Indications, HBV screening bloods and outcomes were collected using electronic records (Allscripts,

\begin{tabular}{|c|c|c|c|c|c|c|}
\hline \multirow[t]{2}{*}{$\begin{array}{l}\text { Time } \\
\text { period }\end{array}$} & \multirow[t]{2}{*}{$\begin{array}{l}\text { Total number } \\
\text { patients (n) }\end{array}$} & \multirow[t]{2}{*}{$\begin{array}{l}\text { Gender } \\
(\%)\end{array}$} & \multicolumn{2}{|c|}{$\begin{array}{l}\text { Serum virology } \\
\text { pre-initiation } \\
\text { screening }\end{array}$} & \multicolumn{2}{|c|}{$\begin{array}{l}\text { Positive virology } \\
\text { detected in } \\
\text { patients screened }\end{array}$} \\
\hline & & & HBSAg & $H B c A b$ & HBSAg & HBcAb \\
\hline $\begin{array}{l}10 / 15- \\
09 / 16\end{array}$ & 161 & $\begin{array}{l}M-59 \\
F-41\end{array}$ & $\begin{array}{l}62 \% \\
(n=100)\end{array}$ & $\begin{array}{l}59 \% \\
(n=95)\end{array}$ & $0 \%$ & $\begin{array}{l}2 \% \\
(n=2)\end{array}$ \\
\hline $\begin{array}{l}10 / 16- \\
09 / 17\end{array}$ & 121 & $\begin{array}{l}M-48 \\
F-52\end{array}$ & $\begin{array}{l}73 \% \\
(n=88)\end{array}$ & $\begin{array}{l}62 \% \\
(n=75)\end{array}$ & $0 \%$ & $\begin{array}{l}4 \% \\
(n=3)\end{array}$ \\
\hline $\begin{array}{l}10 / 17- \\
09 / 18\end{array}$ & 103 & $\begin{array}{l}M-37 \\
F-63\end{array}$ & $\begin{array}{l}92 \% \\
(n=95)\end{array}$ & $\begin{array}{l}73 \% \\
(n=75)\end{array}$ & $0 \%$ & $0 \%$ \\
\hline $\begin{array}{l}10 / 18- \\
09 / 19\end{array}$ & 115 & $\begin{array}{l}M-47 \\
F-53\end{array}$ & $\begin{array}{l}94 \% \\
(n=108)\end{array}$ & $\begin{array}{l}83 \% \\
(n=96)\end{array}$ & $\begin{array}{l}<1 \% \\
(n=1)\end{array}$ & $\begin{array}{l}7 \% \\
(n=7)\end{array}$ \\
\hline $\begin{array}{l}10 / 19- \\
09 / 20\end{array}$ & 106 & $\begin{array}{l}M-46 \\
F-54\end{array}$ & $\begin{array}{l}94 \% \\
(n=100)\end{array}$ & $\begin{array}{l}84 \% \\
(n=89)\end{array}$ & $0 \%$ & $\begin{array}{l}7 \% \\
(n=6)\end{array}$ \\
\hline
\end{tabular}

Telepath). Patients with incomplete or missing records were excluded.

Results A total of 870 patients were included in this study. Seventy percent $(n=611)$ were for oncohaematological indications and $30 \%(\mathrm{n}=258)$ for rheumatological. There were 606 patients newly initiated on Rituximab during the study period (Table 1). HBV screening improved from $62 \%$ to $94 \%$ during the study period.

Eighteen patients were positive or equivocal for Hepatitis B core antibody and 1 for Hepatitis B Surface Antigen. Four patients were prescribed lamivudine prophylaxis, 2 Tenofovir and 1 entecavir. The other patients had no treatment or monitoring. Only 1 case was on adequate length of prophylaxis after being referred to the hepatobiliary service. There were no cases of hepatitis B reactivation in the study period.

Conclusions A significant number of patients are not being screened for Hepatitis B core antibodies, despite local education programmes in 2016 and 2018. Low background rates of $\mathrm{HBV}$ in the local community may account for low levels of previous and current HBV infections. Education of prescribers, modifications to the initiation pharmacy checklists and simplification of computer request process should help to improve Hepatitis B screening in our patients and avoid potentially life-threatening reactivation.

\section{PWE-34 EVALUATING THE NON-VIRAL LIVER DISEASE BURDEN IN PEOPLE LIVING WITH HIV AND ABNORMAL LIVER ENZYMES}

${ }^{1}$ Dev Katarey*, ${ }^{2}$ Yishi Tan, ${ }^{2}$ Harriet Sharp, ${ }^{2}$ Laura Vickers, ${ }^{2}$ Bethany Parnell, ${ }^{1}$ Jonathan Potts ${ }^{2}$ Yvonne Gilleece, ${ }^{2}$ Sumita Verma. ${ }^{1}$ Royal Free Hospital, London, UK; ${ }^{2}$ Brighton and Sussex University Hospitals Trust, Brighton, UK

\subsection{6/gutjnl-2021-BSG.224}

Introduction Liver disease in the absence of viral hepatitis coinfection is a growing problem in people living with HIV (PLWH). Likely contributors include alcohol excess, metabolic syndrome (MS), and hepatotoxic antiretrovirals (ARV). We aimed to assess the prevalence of hepatic fibrosis and associated risk factors in HIV mono-infected individuals with abnormal liver tests.

Methods PLWH with persistently elevated alanine aminotransferase (ALT) for $>6$ months were prospectively assessed using transient elastography, AUDIT questionnaire, and screening for MS. Thresholds for clinically significant hepatic fibrosis (CSHF) and cirrhosis were $>7.1 \mathrm{kPa}$ and $>12.5 \mathrm{kPa}$ respectively, and hepatic steatosis (HS) by a controlled attenuation parameter $(\mathrm{CAP})>237 \mathrm{~dB} / \mathrm{m}$.

Results Of 274 recruited individuals the median age was $52 \mathrm{yrs}$ (IQR 45-59), 93\% men, median HIV duration 15yrs (IQR 1020 ), and undetectable viral load in $96 \%$. Overall, HS was seen in $169(61.7 \%)$ and CSHF was seen in $54(19.7 \%)$, of whom 19 (35.2\%) had cirrhosis and 40 (74.1\%) had HS. Alcohol, MS and ARV were implicated in 24 (44.4\%), 31 $(57.4 \%)$, and $15(27.8 \%)$ of patients with CSHF, respectively. No risk factors were identified in $10(18.5 \%)$ with CSHF. In those with CSHF $(n=54)$ versus those without $(n=220)$, no significant differences were seen in baseline demographic or metabolic co-factors, nor ARV use. On binary and multinomial logistic regression lower HDL cholesterol (HR 0.255, 95\% CI $0.103-0.629, P=0.003$ ) and diabetes (HR 2.558, 95\% CI $1.175-5.571, P=0.018)$ were independent predictors of CSHF. Both FIB-4 and APRI performed poorly in identifying CSHF 